\title{
Fifteen years of successful spread of Salmonella enterica serovar Mbandaka clone ST413 in Poland and its public health consequences
}

\author{
Andrzej Hoszowski', Magdalena Zając ${ }^{1}$, Anna Lalak', Paweł Przemyk², Dariusz Wasyl ${ }^{1}$ \\ ${ }^{1}$ Department of Microbiology, National Veterinary Research Institute, Puławy, Poland \\ ${ }^{2}$ District Sanitary and Epidemiological Station, Bielsko-Biała, Poland
}

Hoszowski A, Zając M, Lalak A, Przemyk P, Wasyl D. Fifteen years of successful spread of Salmonella enterica serovar Mbandaka clone ST413 in Poland and its public health consequences. Ann Agric Environ Med. 2016; 23(2): 237-241. doi: 10.5604/12321966.1203883

\begin{abstract}
In the 1990s, Salmonella enterica serovar (S.) Mbandaka occurred in feed and poultry in Poland. In the following years, the serovar also gained epidemiological importance in other EU countries. The objectives of current study were to evaluate the genetic relationship of contemporary S. Mbandaka with isolates originating from the beginning of the epidemics, and to assess the contribution of poultry as the source of infections in humans. Seventy S. Mbandaka isolated mainly in 2009 - 2010 from humans, poultry, food, and feed were typed with API ID32 ${ }^{\circ}$, MIC, plasmid profiling, PFGE, and MLST. PCR and sequencing were used to identify plasmid mediated quinolone and cephalosporin resistance mechanisms. Six biochemical profiles were identified and 59 of $S$. Mbandaka proved to be susceptible to the applied antimicrobials. Eight strains carried plasmids and a few of them were positive for bla $a_{\mathrm{CMY}-2}$ and qnrS1 genes. Two clusters of $15 \mathrm{Xbal}$-PFGE profiles with similarity of $77.5 \%$ were found. The first cluster, gathered 7 profiles involving historical isolates and several contemporary non-human $\mathrm{S}$. Mbandaka. The predominant profile in the second cluster consisted of 28 human and 1 broiler isolate. MLST analysis showed sequence type ST413 occurring among all tested isolates. The identification of close genetic relationships between S. Mbandaka of human and poultry origin indicates animals as a primal human infection route. Despite Salmonella control programmes, the S. Mbandaka ST413 clone has been circulating for several years in Poland. Salmonella control polices in food production chain should be continuously updated to target serovars of major epidemiological importance. Resistance noted in S. Mbandaka to such antimicrobials as fluoroquinolones and cephalosporins may hinder public health.
\end{abstract}

\section{- Key words}

salmonellosis, epidemiology, PFGE, MLST, plasmids, antimicrobial resistance

\section{INTRODUCTION}

Salmonella enterica subsp. enterica serovar (S.) Mbandaka was firstly isolated from human salmonellosis in the Belgiun Congo in 1948. Soon after, S. Mbandaka occurred in Sweden, Belgium, the Netherlands and Germany, although its epidemiological significance was limited [1]. Finally, $S$. Mbandaka became widespread globally, being currently classified as one of the top-10 serovars responsible for salmonellosis cases in humans in the EU [2]. It was also ranked amongst the 20 most frequent serovars in humans in other European countries, as well as Israel, North America, Africa and New Zealand [3,4]. Since its occurrence in humans in 1997, S. Mbandaka represented one out of the 10 most predominant serovars in Poland, with the second position occurring at $8.79 \%$ in 2010 [5]. During the same period, it became the $8^{\text {th }}$ most common serotype in the EU, with huge increase compared to 2009. In Poland, a high morbidity rate due to $S$. Mbandaka was found in the Silesian province, among citizens living in rural areas close to poultry farms where $S$. Mbandaka was responsible for $32 \%$ all salmonellosis cases in the region in 2010 [6].

$S$. Mbandaka has also gained epidemiological importance for animals and it has been reported as the $5^{\text {th }}$ most frequently noted serovar in both cattle and poultry in the EU, with

Address for correspondence: Andrzej Hoszowski Department of Microbiology, National Veterinary Research Institute, Partyzantów 57, 24-100 Puławy, Poland

E-mail: ahosz@piwet.pulawy.pl

Received: 12 December 2013; accepted: 01 April 2014 variable prevalence in different countries [2], with the top serovars observed elsewhere, both in animals, feeds and foods including dairy products, fish and seafood, cereals, vegetables and fruits, pastries and sweets, and in the environment [3, 4]. Beginning in 1996, S. Mbandaka has been continuously isolated in Poland in veterinary sources such as hens, turkey, pigs, geese, ducks, food and animal feed.

\section{OBJECTIVE}

Taking the above into consideration, the objectives of current study were to asses the possible contribution of poultry as the source of an increasing number of salmonellosis in humans in the Silesian province, and evaluation of the genetic relationship between the contemporary isolates with $S$. Mbandaka originating from the beginning of the epidemics in the mid-1990s.

\section{MATERIALS AND METHOD}

Bacterial isolates. Seventy $S$. Mbandaka were selected for the study: 32 human and 38 non-human isolates, including 2 historical ones. Human isolates originated mostly from patients in the Silesian province $(\mathrm{N}=31)$, including diarrheal $(\mathrm{N}=5)$ and convalescents $(\mathrm{N}=2)$. The remaining isolates were obtained from subclinical faecal carriage $(\mathrm{N}=22)$ or contact cases $(\mathrm{N}=2)$. One isolate was 


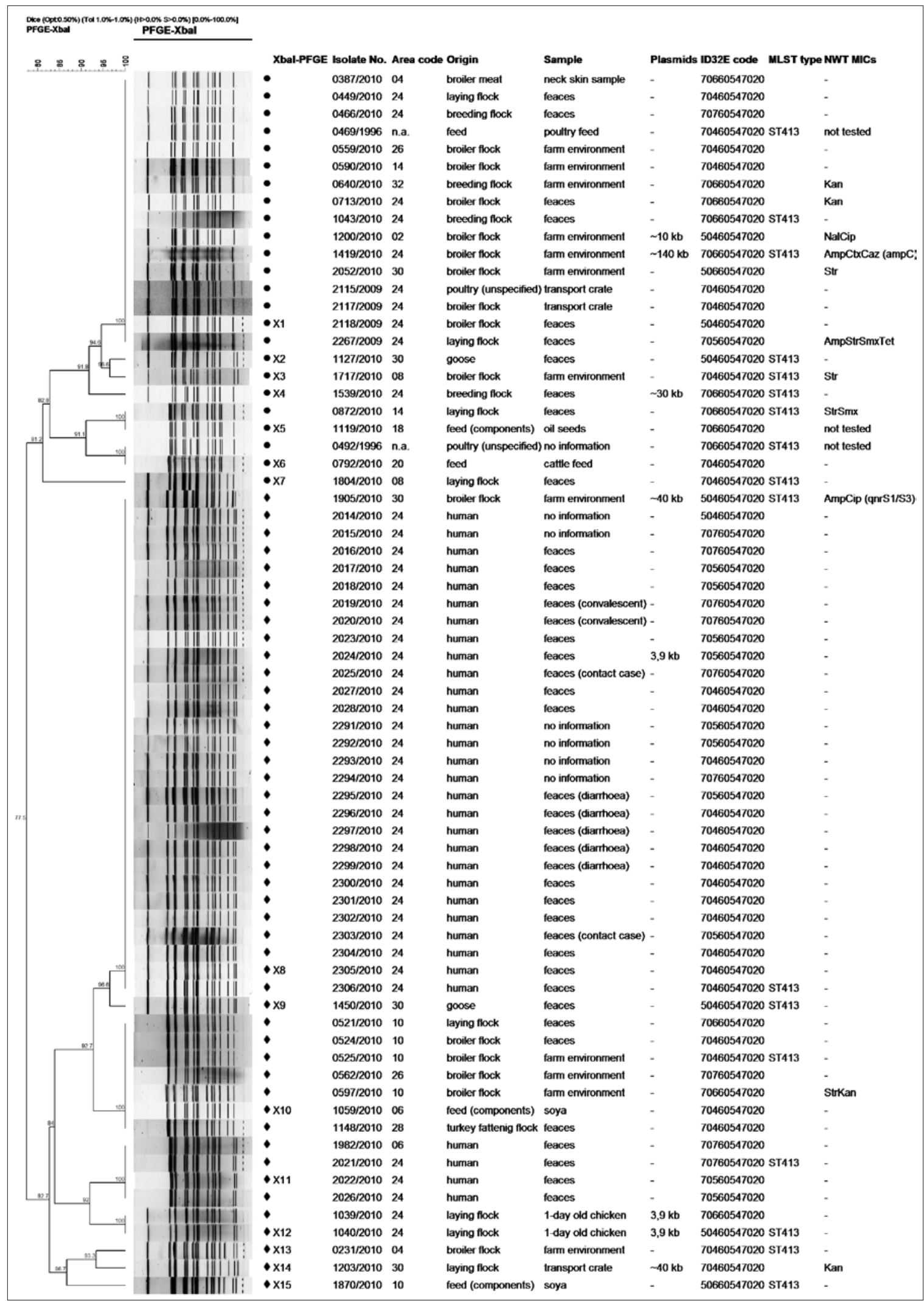

Figure 1. Phylogenetic similarity of $\mathrm{S}$. Mbandaka $(\mathrm{N}=70)$.

Xbal-PFGE cluster I and II marked as $\bullet$ and $\bullet$, respectively. Four last digits of isolate No. designate year of isolation. Area codes: NUTS-2 codes for provinces: 02 - dolnośląskie, (Lower Silesia) 04-kujawsko-pomorskie, 06 - lubelskie, 08 - lubuskie, 10 - łódzkie, 14-mazowieckie 18 - podkarpackie, 20 - podlaskie, 24 - śląskie (Silesia), 26 - świętokrzyskie, 28 - warmińsko-mazurskie, 30 - wielkopolskie, 32 - zachodniopomorskie.

Resistance profiles (non-wild type MICs - NWT): antimicrobial abbreviations and minimal inhibitory concentration epidemiological cut-off values (EUCAST; NWT > mg/L): Amp - ampicillin ( $8 \mathrm{mg} / \mathrm{L}), \mathrm{Ctx}$ - cefotaxime $(0,5 \mathrm{mg} / \mathrm{L}), \mathrm{Caz}$ - ceftazidime (2mg/L), Cip - ciprofloxacin (0.064 mg/L), Kan kanamycin (4 mg/L), Nal - nalidixic acid (16 mg/L), Str - streptomycin (16 mg/L), Smx - sulfametoxazole (256 mg/L), Tet - tetracycline (8 mg/L); “-“ designates wild type MICs (WT) for the above-mentioned antimicrobials as well as colistin ( $2 \mathrm{mg} / \mathrm{L})$, chloramphenicol ( $16 \mathrm{mg} / \mathrm{L})$, florfenicol ( $16 \mathrm{mg} / \mathrm{L})$, gentamycin ( $2 \mathrm{mg} / \mathrm{L})$, and trimethoprim (2 mg/L). 
recovered from a human faecal carrier living in the Lublin province. The non-human study group $(\mathrm{N}=36)$ was gathered in 2009 - 2010 from asymptomatic poultry flocks $(\mathrm{N}=32)$, broiler meat $(\mathrm{N}=1)$, or feed $(\mathrm{N}=3)$. To ascertain the widest possible diversity of the isolates they were selected based on animal species and production type, date and place of isolation. The adopted inclusion criteria guaranteed the variety of non-human isolates (Simpson index of diversity reached 0.785). Additionally, 2 isolates found in 1996 in poultry and feed and presenting the most frequent historical clonal lineage A, were included in the study [7] (Fig. 1).

Identification of isolates. Serotyping according to WhiteKauffmann-Le Minor scheme [8] was performed to verify Salmonella serovar. Biochemical properties of isolates were evaluated by API ID $32^{\circ}$, performed and coded according to manufacturer's instruction (bioMérieux Polska, Warsaw, Poland).

MIC testing and identification of selected resistance mechanisms. Microbiological resistance was assessed by the microbroth dilution method. Minimal Inhibitory Concentration (MIC) of 14 antimicrobials (Fig. 1) were interpreted according to the epidemiological cut-off values (http://www.eucast.org). Etests ${ }^{\circledast}$ (ESBL and AmpC; AB bioMerieux, Solna, Sweden) were performed for phenotypic confirmation of cephalosporin resistance and differentiation between extended spectrum betalactamase and AmpC cephalosporinase production. Resistance mechanism was identified with bla ${ }_{\mathrm{CMY}-2}$ PCR amplification [9]. Isolate showing wild-type nalidixic acid MIC and microbiological resistance to ciprofloxacin was tested with PCR for qnrS amplicon [10], and further sequenced for resistance mechanism identification (Oligo, Institute of Biochemistry and Biophysics, Polish Academy of Sciences, Warsaw, Poland). Both PCR assays were performed with Maxima Hot Start PCR Master Mix according to manufacturer's recommendation (Fermentas Life Sciences, Thermo Scientific, Lithuania).

Molecular characterization. Plasmid profiling was performed with previously described lysis by the alkali method [7]. Plasmidic DNA was run in $1.0 \%$ agarose gel against $E$. coli 39R and V517 molecular size markers and interpreted with BioNumerics (version 5.1, Applied Maths, Sint-MartensLatem, Belgium). Pulsed field gel electrophoresis (PFGE) was carried out according to the PulseNet protocol following DNA digestion with $X b a \mathrm{I}$, as described earlier $[11,12]$. Additionally, SpeI-PFGE was carried out on a subset of isolates $(\mathrm{N}=55)$ representing predominant XbaI-PFGE profiles. BioNumerics fingerprint analysis included UPGMA, Dice coefficient,
$0.5 \%$ optimisation, and $1.5 \%$ position tolerance. Multilocus sequence typing (MLST) was performed on 18 isolates representing most of the identified XbaI-PFGE patterns [13].

\section{RESULTS}

After biochemical and serological testing, all isolates were identified as $S$. Mbandaka. ID32E biotyping distinguished 6 biochemical profiles with different ability for decomposition of arginine and 5-ketogluconian and production of lipase and a-glucosidase (Tab. 1). Human isolates were classified to 4 profiles and they were predominant in biotype 1 and 4 . Two of the 6 profiles covered only $S$. Mbandaka originating from non-human sources. Simpson's diversity index for biotyping achieved the value of 0.766 .

Most of tested $S$. Mbandaka $(\mathrm{N}=59)$ proved to be susceptible (wild-type) to the applied antimicrobials. Eight resistance profiles were observed in 11 isolates showing nonwild type MICs to one (Str or Kan) up to 4 compounds (AmpStrSmxTet). Most often, the resistance profiles were registered in broilers isolates (7/15), layers (3/8), or those recovered from breeders (1/4), but not from humans (Fig. 1).

Eight out of 70 tested strains carried plasmids with molecular mass ranging from $3.9 \mathrm{~kb}-140 \mathrm{~kb}$ (5 plasmid profiles). Four of them showed NWT MICs up to 3 tested antimicrobials. Cephalosporin NWT isolate carrying $\sim 140 \mathrm{~kb}$ plasmid exhibit synergetic effect in AmpC Etest and was found positive for $b l a_{\mathrm{CMY}-2}$ specific amplicon. Another $S$. Mbandaka of ampicillin and ciprofloxacin NWT MIC carried $40 \mathrm{~kb}$ plasmid with qnrS1/S3 gene (Fig. 1).

Two clusters composed of $15 \mathrm{XbaI}$-PFGE profiles with a similarity of $77.5 \%$ were found (Fig. 1). The first cluster of 7 profiles with a similarity of $81.2 \%$ involved 2 isolates from 1996 , and several contemporary non-human S. Mbandaka. The predominant $\mathrm{XbaI}$-PFGE SMb-X1 profile covered 16 isolates originating from poultry breeding $(\mathrm{N}=3)$, broiler $(\mathrm{N}=8)$, or laying $(\mathrm{N}=2)$ flocks as well as single $S$. Mbandaka from broiler meat and historical feed isolate. The remaining profiles from this cluster $(\mathrm{SMb}-\mathrm{X} 2 \div \mathrm{SMb}-\mathrm{X} 7)$ gathered isolates from geese, laying hens and broilers, as well as an historical unspecified poultry source and those contemporarily obtained from feed with a similarity of $91.1 \%$.

Cluster II gathered 8 XbaI-PFGE profiles. The predominant $\mathrm{SMb}-\mathrm{X} 8$ consisted of 28 human isolates and were indistinguishable from broiler isolate (1905/2010). It showed close similarity (up to $92.7 \%$ ) to the following 2 profiles (SMb-X9 and $\mathrm{SMb}-\mathrm{X} 10$ ) gathering isolates from different animal species and feed. Similar genetic diversity was noted between SMb-X11 (4 human isolates) and SMb-X12

Table 1. Biochemical profiles in tested $S$. Mbandaka isolates $(N=70)$

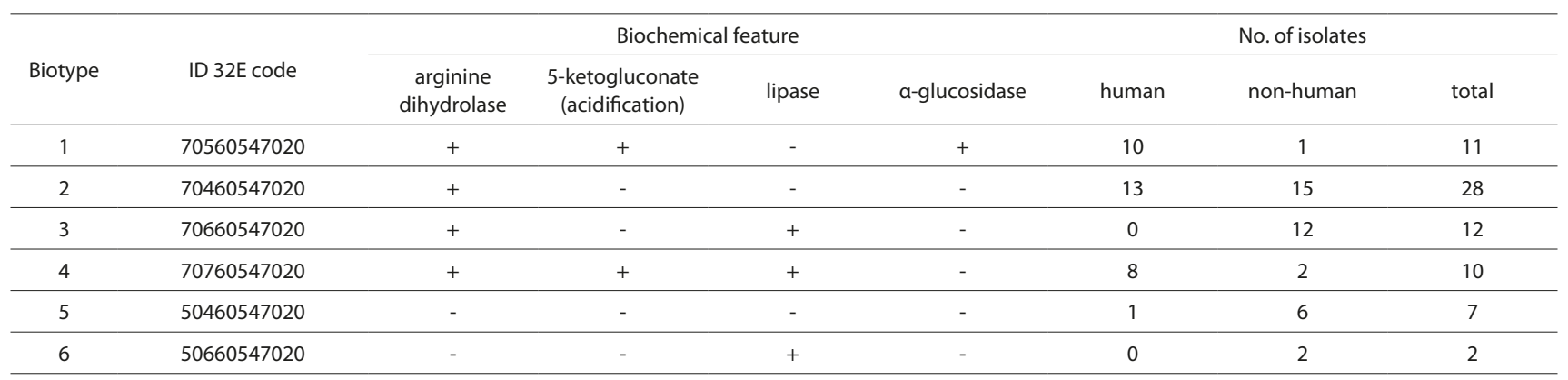


(S. Mbandaka from egg laying flocks). Each of the remaining profiles (SMb-X13, -X14, and -X15) gathered single isolates from broilers, laying hens or feed with $84.0 \%$ similarity to other cluster II profiles. Simpson's diversity index for XbaI-PFGE typing reached 0.770. SpeI-PFGE, with a few exceptions, did not further discriminate isolates belonging to the same XbaI profile (data not shown).

MLST analysis showed sequence type ST413 occurring among all tested isolates (Fig. 1).

\section{DISCUSSION}

Since its introduction in the mid-1990s-[7], S. Mbandaka has gained some epidemiological importance, being responsible for infections in animals and humans as well as contaminations of food and feed, both in Poland and the EU $[2,5]$. In Poland, the presented results confirm that it was due to a single clone of Mbandaka ST413 introduced in 1996, and circulating to- date between feed, poultry, foods, and humans. The reasons for the epidemiological success of this particular clonal lineage might be hypothesized based on recent studies on biofilm production, pathogenicity, and host adaptation [14]. The observed variability of PFGE, plasmid, biochemical, and resistance profiles of $S$. Mbandaka proves that the on-going epidemics in Poland results from multiple genetic rearrangements during transmission routes involving feed, various poultry, food of animal origin, and finally reaching humans.

Numerous molecular and phenotypic patterns were observed, PFGE profiles comprising most of human isolates (in the case of SMb-X8 also broiler isolate No.1905/2010) and a high similarity found between profiles embracing isolates from layers, broilers, turkeys, and goose with human profiles indicated poultry as a plausible source of human infection. Although direct contact with poultry was identified as the source of a mult-State outbreak of $S$. Mbandaka in the UAS [15], the relation of increasing number of salmonellosis cases in the Silesian province [6] has not been confirmed with $S$. Mbandaka infected poultry farms in the region. The finding of indistinguishable human isolates with the one from broilers originating from distinct province, pinpoints the role of contaminated food being transferred to the countrywide market. Since the presented study focused on $S$. Mbandaka cases in a narrow time window and occurred mostly in a single province of Poland, similar scenarios may happen elsewhere involving the described poultry-related strains [7]. Similarly, the Austrian Reference Centre for Salmonella also noticed an increase in the number of salmonellosis due to $S$. Mbandaka in 2010, and the investigation revealed eggs or egg dishes obtained from laying flocks fed with contaminated commercial feed as responsible for the outbreak [16]. However, human infections due to $S$. Mbandaka could not always be linked to animals and food of animal origin. For example, several cases reported from New Zealand were related to sesame seeds paste [17]. It is intriguing why $S$. Mbandaka is still being isolated from flocks despite the implementation of Salmonella control programmes that require enhanced bio-security, including the cleaning and disinfection of the poultry farm environment. One of the possible explanations is that it is probable that the disinfection process is not fully effective, and the pathogen, as well other Salmonella, can still circulate within flocks and between different production sectors. Additionally, S. Mbandaka might also develop biofilms protecting bacteria against disinfectants [18]. This was one of the most prevalent serovar found in airborne dust sampled from layer farms in Japan, which undoubtedly helped the circulation within poultry flocks and hampering the elimination of this pathogen from the farm environment during disinfection [19]. It is noteworthy that the difficult eradication of $S$. Mbandaka from poultry production is further limited by control programmes targeting selected serovars, and the decrease of their prevalence might be compensated by the others gaining some epidemiological importance.

Similar to other studies [20, 21], S. Mbandaka isolated from various sources in Poland over several years remained susceptible to antimicrobials. The discovery of a few resistances in $S$. Mbandaka proved that the phenomenon is not a prerequisite for the above-presented epidemiological success of the serovar, despite antimicrobial selection pressure in Polish poultry production [22]. The high resistance rates observed in S. Mbandaka from layer farms in Japan [19] or humans in Morocco [23] might have been related with the dissemination of bacterial clones showing specific resistance patterns, as observed in Poland for S. Kentucky [11] or $S$. Typhimurium [12].

Attention should bedrawn to plasmid-mediated mechanisms encoding cephalosporin and fluoroquinolone resistance antimicrobial classes considered critically important for public health [24]. AmpC-type cephalosporinase CMY-2 gene encoded on a heavy weight plasmid of approximately $140 \mathrm{~kb}$ has been previously noted in clinical isolates in Tunisia [25]. Plasmid-mediated $q n r S 1 / S 3$ found in other poultry isolates is the commonest PMQR mechanisms observed in Salmonella and E. coli in Poland (unpublished date) [10]). Both mechanisms have to be considered a serious health threat resulting from antimicrobial selection pressure and horizontal spread involving non-pathogenic bacteria $[9,10]$.

\section{CONCLUSIONS}

Summing up, the identification of close genetic relationships between $S$. Mbandaka of human and poultry origin indicates animals and food as primal human infection routes, but not close contact with neighbouring poultry farms. The tremendous epidemiological success of S. Mbandaka ST413 clone was confirmed. Despite, or partly due to Salmonella control programmes implemented in poultry production, the clone has been circulating for several years between poultry, feeds, and foods, finally compromising public health. It is successive proof that Salmonella control polices in the food production chain should be continuously updated to target serovars of major epidemiological importance to enhance efficient consumer protection. That protection is further hampered with the ecologically sound phenomenon of selection and horizontal spread of resistance to critically important antimicrobials: fluoroquinolones and cephalosporins.

\section{REFERENCES}

1. Kelterborn E. Salmonella species. First isolations, names, and occurrence. Leipzig: S. Hirzel Verlag; 1967. 
2. European Food Safety Authority, European Centre for Disease Prevention and Control. The European Union summary report on trends and sources of zoonoses, zoonotic agents and food-borne outbreaks in 2011. EFSA Journal. 2013; 11(4): 1-255.

3. Hendriksen RS. Global epidemiology of non-typhoidal Salmonella infections in humans. Søborg: Technical University of Denmark; 2010.

4. Ben Aissa R, Al-Gallas N, Troudi H, Belhadj N, Belhadj A. Trends in Salmonella enterica serotypes isolated from human, food, animal, and environment in Tunisia, 1994-2004. J Infect. 2007; 55(4): 324-339.

5. Państwowy Zakład Higieny, Główny Inspektor Sanitarny. Choroby zakaźne i zatrucia w Polsce w 2011 r. Warszawa, 2012.

6. Wałga T, Hudzik G, Cieślik-Tarkota R. Diseases induced by Salmonella Mbandaka in Silesian Province - new risk to public health? Proceedings of the Epidemiological threats for human health; 20 September 2010 Puławy, Poland; Państwowy Instytut Weterynaryjny - Państwowy Instytut Badawczy, 2010.

7. Hoszowski A, Wasyl D. Typing of Salmonella enterica subsp. enterica serovar Mbandaka isolates. Vet Microbiol. 2001; 80(2): 139-148.

8. Grimont PAD, Weill F-X. Antigenic formulas of Salmonella serovars. 9th editon ed. Paris: WHO Collaborating Centre for Research on Salmonella, Institute Pasteur; 2007.

9. Wasyl D, Hasman H, Cavaco LM, Aarestrup FM. Prevalence and characterization of cephalosporin resistance in nonpathogenic Escherichia coli from food-producing animals slaughtered in Poland. Microb Drug Resist. 2012; 18(1): 79-82.

10. Veldman K, Cavaco LM, Mevius D, Battisti A, Franco A, Botteldoorn $\mathrm{N}$, et al. International collaborative study on the occurrence of plasmidmediated quinolone resistance in Salmonella enterica and Escherichia coli isolated from animals, humans, food and the environment in 13 European countries. J Antimicrob Chemother. 2011; 66(6): 1278-1286.

11. Wasyl D, Hoszowski A. First isolation of ESBL-producing Salmonella and emergence of multiresistant Salmonella Kentucky in turkey in Poland. Food Res Int. 2012; 45(2): 958-961.

12. Wasyl D, Hoszowski A. Occurrence and characterization of monophasic Salmonella enterica serovar Typhimurium $(1,4,[5], 12: \mathrm{i}:-)$ of non-human origin in Poland. Foodborne Pathog Dis. 2012; 9(11): 1037-1043.

13. University College Cork (UCC). MLST Databases at the Environmental Research Institute. http://mlst.ucc.ie/.

14. Hayward MR, Jansen V, Woodward MJ. Comparative genomics of Salmonella enterica serovars Derby and Mbandaka, two prevalent serovars associated with different livestock species in the UK. BMC Genomics. 2013; 14: 365

15. Multistate outbreak of human Salmonella infections linked to live poultry. Centers for Disease Control and Prevention, http://www.cdc. gov/salmonella/live-poultry-04-13/index.html. (access: 2013.10.14).

16. Allerberger F. Molecular typing in public health laboratories: from an academic indulgence to an infection control imperative. J Prev Med Public Health. 2012; 45(1): 1-7.

17. Public Health Surveillance. Human Salmonella Isolates. Information for New Zealand Public Health Action, 2012, No 12. 2013.

18. Marin C, Hernandiz A, Lainez M. Biofilm development capacity of Salmonella strains isolated in poultry risk factors and their resistance against disinfectants. Poult Sci. 2009; 88(2): 424-431.

19. Iwabuchi E, Maruyama N, Hara A, Nishimura M, Muramatsu M, Ochiai T, et al. Nationwide survey of Salmonella prevalence in environmental dust from layer farms in Japan. J Food Prot. 2010; 73(11): 1993-2000.

20. Filioussis G, Petridou E, Johansson A, Christodoulopoulos G, Kritas SK. Antimicrobial susceptibility and genetic relatedness of Salmonella enterica subsp enterica serovar Mbandaka strains, isolated from a swine finishing farm in Greece. Afr J Microbiol Res. 2008; 2(11): 313-315.

21. Deckert A, Gow S, Rosengren L, Leger D, Avery B, Daignault D, et al. Canadian integrated program for antimicrobial resistance surveillance (CIPARS) farm program: results from finisher pig surveillance. Zoonoses Public Health. 2010; 57(Suppl 1): 71-84.

22. Wasyl D, Hoszowski A, Zając M, Szulowski K. Antimicrobial resistance in commensal Escherichia coli isolated from animals at slaughter. Front Microbiol. 2013; 4: 221.

23. Ammari S, Laglaoui A, En-Nanei L, Bertrand S, Wildemauwe C, Barrijal $S$, et al. Isolation, drug resistance and molecular characterization of Salmonella isolates in northern Morocco. J Infect Dev Ctries. 2009; 3(1): 41-49.

24. Collignon P, Powers JH, Chiller TM, Aidara-Kane A, Aarestrup FM. World Health Organization ranking of antimicrobials according to their importance in human medicine: A critical step for developing risk management strategies for the use of antimicrobials in food production animals. Clin Infect Dis. 2009; 49(1): 132-141.

25. Makanera A, Arlet G, Gautier V, Manai M. Molecular epidemiology and characterization of plasmid-encoded beta-lactamases produced by Tunisian clinical isolates of Salmonella enterica serotype Mbandaka resistant to broad-spectrum cephalosporins. J Clin Microbiol. 2003; 41(7): 2940-2945. 